\title{
Physician Ratings Published on Healthcare Organizations' Websites: Are They Biased?
}

\author{
Nima Kordzadeh \\ Worcester Polytechnic Institute \\ nkordzadeh@wpi.edu
}

\begin{abstract}
In today's age of social media, individuals use physician-rating websites (PRWs) to find information about healthcare providers and make decisions on which providers to choose accordingly. In line with this trend, healthcare organizations such as clinics and hospitals offer their own physician-rating platforms and mechanisms. However, a major concern regarding this form of privately-administered rating mechanism is the potentially high level of bias that may make the ratings published on those websites inaccurate and unreliable. In this study, we examined this form of bias. We used two hospital websites and four independent PRWs including RateMDs, HealthGrades, Vitals, and Google Reviews to collect, compare, and analyze patient satisfaction scores associated with a total of 569 physicians working in two hospitals located in Utah. The results of the analysis of variance (ANOVA), paired t-tests, and box plots demonstrated that, as hypothesized, the ratings published on the hospitals' websites had significantly higher mean values and narrower distributions than those published on the independent PRWs. Our findings offer important implications for research and practice.
\end{abstract}

\section{Introduction}

Physician-rating websites (PRWs) have become a major social media platform through which Internet users evaluate the quality of care provided by physicians, dentists, clinics, hospitals, and other entities in the context of healthcare [15]. The information shared on PRWs are typically in the forms of numerical ratings and narrative comments. This information can help individuals make more informed decisions on which healthcare providers to choose [9]. Nearly $60 \%$ of the respondents to a nationally representative survey conducted in 2012 reported that PRWs were important to them when selecting a primary care doctor [10]. The results of a similar survey study conducted in Germany in 2012 revealed that $29.3 \%$ of the 986 respondents were aware of the existence of PRWs and $26.1 \%$ of the respondents had used at least one of those websites [26]. PRWs have also been widely adopted in other countries such as Canada [20] and China [11].

RateMDs.com, for example, is one of the major PRWs based in the United States. As of April 2017, this website had more than 2.6 million reviews about healthcare providers. RateMDs.com lets users rate healthcare organizations such as clinics and hospitals based on cleanliness, facilities, services, and value. Furthermore, users can use this website to rate physicians on different criteria including staff, helpfulness, punctuality, and knowledge and also post anonymous comments about healthcare providers. Vitals.com is another popular PRW in the United States. As of April 2017, Vitals.com offered more than 7.8 million ratings and reviews of doctors, specialists, and treatment and recovery facilities gathered from thousands of users.

More recently, healthcare organizations have started offering their own physician-rating platforms. This is in line with the social media strategies that healthcare organizations have recently embarked on [16]. In 2012, the University of Utah Health Care started posting the results of patient surveys on the hospital's website [23]. They did so because, according to their senior director of interactive marketing and web, they "knew [their] patient satisfaction scores were really strong and [they] had a good story to share with [their] patients" [23]. Several other hospital systems have followed this trend over the past few years and started posting patient ratings and comments about their own doctors on their websites. Northwell Health (formerly North ShoreLIJ) [24], University of Pittsburg Medical Center [13], and Cleveland Clinic [21] are among the healthcare organizations that have voluntarily shared their physician ratings on their websites.

A major characteristic of the PRWs that are offered by healthcare organizations is that the administrators of those websites have a full control over what to be published and what not to be published. For example, on one of the US-based PRWs, it is stated that "we post 
all comments, verbatim, both positive and negative, as long as they aren't deemed to be libelous, don't contain profane or vulgar language and don't compromise patient privacy." ${ }^{1}$ But it is not clearly explained through what mechanisms and based on what procedures, keywords, and criteria a comment would be viewed as libelous and hence, would be removed. As a result, the administrators of the website may prefer, or be instructed, to remove the ratings and comments that convey patients' bad experience and dissatisfaction with healthcare services, treatments, and outcomes. If this form of information filtration occurs, the ratings posted on healthcare organizations' websites will become significantly biased toward positive values. This potential bias can ultimately make those online physician ratings unreliable [12].

Another potential source of bias in the ratings published on healthcare organizations' websites is that in general, patients may not be willing to post negative feedback about their healthcare providers on providers' websites [6]. This can also lead to an artificial increase in the average physician ratings compared to reality and even compared to the corresponding ratings published on independent PRWs such as RateMDs.com and Vitals.com. As a result, the rating information published by healthcare organizations would become not as useful as they are intended to be and might even become misleading to the information seekers.

In order to shed light on this phenomenon, we conduct this preliminary study. Given the critical role of online reviews in today's world, in particular in the context of healthcare, the results of this study will offer important practical implications for Internet users, providers and administrators of PRWs and privatelyadministered patient satisfaction surveys, and policy makers in the realm of virtual health communities. Our findings will also provide theoretical implications in the contexts of online social networks, online health communications, healthcare administration, and social media marketing.

The remainder of this paper is structured as follows. The second section provides a review of the extant literature relevant to this study. The main hypothesis is also presented in that section. The third section explains the research method used in this study. The fourth section presents the results of the different sets of analysis conducted on the dataset. The fifth section provides a discussion on our findings as well as the limitations and future research directions. Finally, the last section presents the conclusions drawn in this study.

\footnotetext{
${ }^{1}$ https://intermountainhealthcare.org/websiteinformation/provider-ratings/
}

\section{Literature Review and Hypothesis}

Prior studies in the context of health social media have examined the characteristics of PRWs $[17,18]$ as well as the characteristics and views of users versus non-users of those websites [12, 26]. For example, Lagu et al. [18] analyzed more than 8,000 reviews on 28 PRWs and found that the average numbers of ratings and comments per physician were significantly different across those websites. Their results also revealed that the PRWs that they investigated were different from one another in terms of advertising models, registration requirements, search functions, physicians' demographics available to the public, and the criteria and sub-criteria based on which patients evaluate the performance of physicians. In a survey of 1,006 randomly selected patients in Germany, Terlutter et al. [26] found that younger people, women, highly-educated individuals, and people with chronic conditions were more prone to use PRWs.

Prior research has also investigated the inherent bias that exists in the information shared on independent PRWs. More specifically, the results of those studies have demonstrated that most patients would give physicians favorable ratings and hence, the ratings of physicians could, in general, be biased toward positive values [14]. Lagu et al. [17] found that $88 \%$ of the online physician ratings were positive; whereas, only six percent were negative. Furthermore, Kadry et al. [14] found that $61.5 \%, 57.74 \%$, and $74 \%$ of the reviews on PRWs that used a 100-point scale, 5point scale, and 4-point scale, respectively, had ratings of $75 \%$ or higher. Similarly, Gao, et al. [6] reported that $45.80 \%$ and $12 \%$ of the physicians rated on RateMDs received the highest and lowest scores, respectively. In another study, Emmert and Meier [4] analyzed more than 127,000 ratings of 53,585 physicians made by 107,148 patients on jameda, the most popular PRW in Germany (according to the authors), and found that nearly $80 \%$ of the reviews belonged to the top two rating levels (based on a 6point scale) and only 3\% of the physicians were rated at the lowest level. More recently, Gao, Greenwood, Agarwal, and McCullough [5] analyzed this phenomenon and demonstrated that patients were more likely to rate and discuss physicians with high patient-perceived quality in online environments. Moreover, their findings showed that patients were more likely to exaggerate their opinions when rating physicians online. 
All in all, the extant literature has consistently shown that online physician reviews and ratings are to some extent biased toward positive values and sentiments. One of the major reasons for this bias, according to the literature, is that patients are generally reluctant to share their negative experiences online when it comes to healthcare services and treatments, especially if they believe their identity could somehow be disclosed in those online rating environments. Hanauer et al. [10] found that 34\% of the participants in that study were concerned about their identity being disclosed in PRWs and 26\% had concerns about the potential actions that physicians could take against them if they rate their physicians negatively. One of those potential actions could be that physicians might not provide the highest quality of care that they could offer to the patients who are known to have left negative comments. Additionally, patients may even get sued by physicians for leaving such negative comments about them in social media environments $[19,25]$.

The risks of sharing negative experiences and feedback on healthcare organizations' websites may be perceived to be higher than doing so on independent PRWs. The reason is that it might be easier for healthcare organizations to recognize the identity of an individual who leaves a comment on their website than of those who leave comments on independent PRWs. This higher level of potential concern may result in a more positive ratings and reviews about physicians on healthcare websites than on independent PRWs. Moreover, healthcare organizations (or the companies hired by them to handle their patient satisfaction surveys) are likely to be able, and willing, to filter ratings and reviews based on the extent to which those patient-generated information are favorable to those organizations and their doctors. This possible information filtration can make the ratings and reviews posted on healthcare organizations' websites even more biased toward positive values and sentiments than those published on independent PRWs. This high level of systematic bias, if empirically demonstrated, could be dangerous as thousands of Internet users may rely on these rating information to make decisions for their healthcare.

Despite the importance of understanding the differences between organization-controlled PRWs and independent PRWs, in particular in terms of information bias, to the best of our knowledge, no study has yet examined this matter. Therefore, in this study, we aim to investigate this phenomenon by analyzing and comparing the rating information published on healthcare organizations' websites versus those published on independent PRWs. More specifically, we hypothesize:
- Physician ratings published on healthcare organizations' websites are significantly more biased toward positive values than their corresponding ratings published on independent PRWs.

\section{Method}

In order to address the hypothesis, we selected one of the major health systems in the United States that has established a hospital-oriented physician rating system. This not-for-profit health system is based in Salt Lake City, Utah, and includes 22 hospitals offering a broad range of clinical services to the patients in that region. According to the hospitals' websites, their physician rating surveys are based on the nationally developed Clinician and Group Consumer Assessment of Healthcare Providers and Systems (CG-CAHPS ${ }^{\circledR}$ ) survey. Ratings are published on the websites for those doctors who have at least 30 surveys completed.

We randomly selected two hospitals operating under that health system (aliased hospital_1 and hospital_2 from this point on in this paper), visited their websites, and collected ratings on 300 and 269 physicians, including medical doctors (MDs), doctors of osteopathic medicine (DOs), and doctors of podiatric medicine (DPMs), who worked in those two hospitals, respectively. These were all the physicians with ratings in those hospitals as of the date of data collection. The ratings were measured in terms of patient satisfaction scores anchored on a 5-point scale from (1) very dissatisfied to (5) very satisfied. Each of the physicians in hopsptal_1 had an average of 152 and 121 ratings and comments, respectively; whereas, these numbers for the physicians in hospital_2 were 182 and 118, respectively. Also, the average satisfaction scores for hospital_1 and hospital_2 were 4.65 and 4.64 , respectively.

In order to compare this information with the corresponding information posted on other PRWs, we collected ratings of the same physicians posted on four independent PRWs including HealthGrades.com, Vitals.com, RateMDs.com, and Google reviews. These websites are among the most widely used PRWs in the United States and have been frequently used in the extant literature (e.g., [1, 3, 5, 18]). However, not all the physicians had ratings on all the websites. Further, in order to improve the reliability of results and reduce random error, we excluded the physicians with less than five ratings on each website from the dataset. Overall satisfaction scores for each website are presented in Table 1, including mean values and standard deviations in parentheses. 
Table 1: Patient satisfaction scores

\begin{tabular}{|l|l|l|}
\hline $\begin{array}{l}\text { Mean value } \\
\text { (Std Dev) }\end{array}$ & $\begin{array}{l}\text { Overall } \\
\text { satisfaction } \\
\text { Hospital_1 }\end{array}$ & $\begin{array}{l}\text { Overall } \\
\text { satisfaction } \\
\text { Hospital_2 }\end{array}$ \\
\hline Hospital & 4.65 & 4.64 \\
website & $(0.13)$ & $(0.12)$ \\
& $\mathrm{N}=300$ & $\mathrm{~N}=269$ \\
\hline Google & 4.20 & 4.56 \\
reviews & $(0.69)$ & $(0.49)$ \\
& $\mathrm{N}=54$ & $\mathrm{~N}=31$ \\
\hline Vitals & 4.11 & 4.21 \\
& $(0.58)$ & $(0.57)$ \\
& $\mathrm{N}=170$ & $\mathrm{~N}=132$ \\
\hline HealthGrades & 3.99 & 3.95 \\
& $(0.65)$ & $(0.66)$ \\
& $\mathrm{N}=225$ & $\mathrm{~N}=172$ \\
\hline RateMDs & 3.80 & 3.83 \\
& $(0.68)$ & $(0.60)$ \\
& $\mathrm{N}=68$ & $\mathrm{~N}=37$ \\
\hline
\end{tabular}

\section{Analysis and Results}

To investigate the potential differences between patient satisfaction scores published on different websites, we first performed two sets of one-way Analysis of Variance (ANOVA), one for each hospital, using JMP statistical software. The initial results of the ANOVA models presented in Tables 2 and 3 as well as Figure 1 demonstrated that there were significant differences between pairs of websites in terms of overall satisfaction scores for each hospital.

Table 2: ANOVA results (hospital_1)

\begin{tabular}{|l|l|l|l|l|}
\hline Source & DF & $\begin{array}{l}\text { Sum of } \\
\text { Squares }\end{array}$ & $\begin{array}{l}\text { Mean } \\
\text { Square }\end{array}$ & F Ratio \\
\hline Model & 4 & 78.48 & 19.62 & $74.92^{* * *}$ \\
\hline Error & 812 & 212.64 & 0.26 & \\
\hline C. Total & 816 & 291.11 & & \\
\hline${ }^{*} p<0.05,{ }^{\star *} p<0.01,{ }^{\star *} p<0.001$ \\
\hline
\end{tabular}

Table 3: ANOVA results (hospital_2)

\begin{tabular}{|l|l|l|l|l|}
\hline Source & DF & $\begin{array}{l}\text { Sum of } \\
\text { Squares }\end{array}$ & $\begin{array}{l}\text { Mean } \\
\text { Square }\end{array}$ & F Ratio \\
\hline Model & 4 & 63.12 & 15.78 & $72.1579 * * *$ \\
\hline Error & 636 & 139.08 & 0.22 & \\
\hline C. Total & 640 & 202.19 & & \\
\hline \multicolumn{2}{|l}{$p<0.05,{ }^{\star \star} p<0.01,{ }^{\star \star \star} p<0.001$} \\
\hline
\end{tabular}

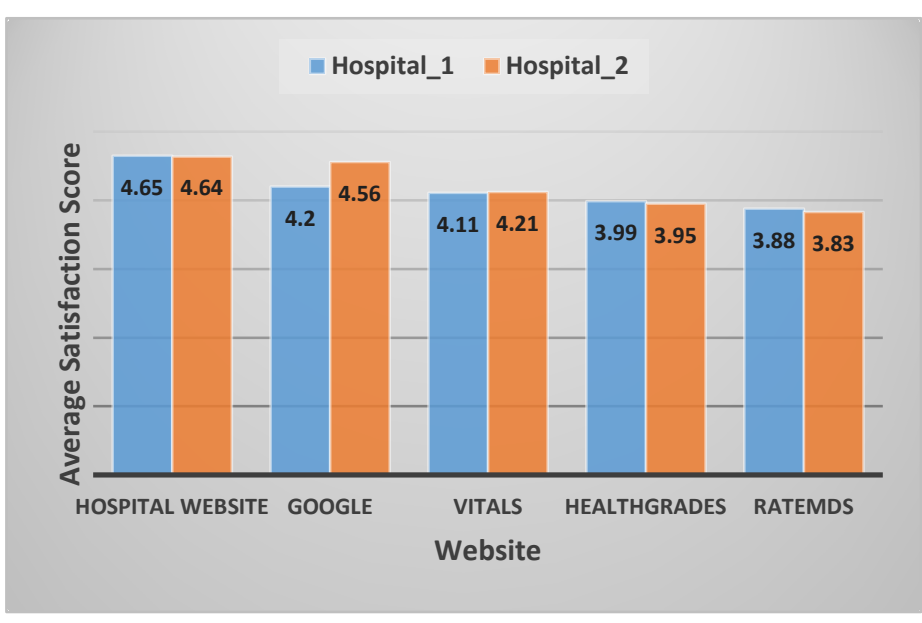

Figure 1: Patient satisfaction scores

To test the hypothesis and better understand the results of the ANOVA models, we conducted a pairwise comparison test, namely Tukey's HSD test. The results are presented in Table 4. Accordingly, for each hospital, the websites that are not tagged with the same letter (i.e., A, B, and C) have significantly different mean values. For example, for hospital_1, the mean values associated with the hospital's website and Vitals are significantly different as they are tagged with different letters ('A' and 'B', respectively); whereas, the mean values for Vitals and HealthGrades are not significantly different as they are both tagged with the letter 'B'.

The results of the Tukey's HSD test demonstrated that for hospital_1, the means of the scores posted on the hospital's website were significantly higher than the means of the scores posted on the other four websites. This conclusion holds for hospital_2 as well, except that the mean of the scores posted on that hospital's website was marginally, but not significantly, greater than the mean of the scores on Google Reviews. Thus, overall, the proposed hypothesis was to a great extent supported.

Also, to further understand the potential differences between the ratings posted on the four independent PRWs, although not directly related to the proposed hypothesis, we examined the Tukey's HSD test results and found that Google Reviews and RateMDs contained the highest and lowest mean scores for the both hospitals, respectively. 
Table 4: Tukey's HSD test results

\begin{tabular}{|l|l|l|}
\hline Website & $\begin{array}{l}\text { Letters for } \\
\text { hospital_1 }\end{array}$ & $\begin{array}{l}\text { Letters for } \\
\text { hospital_2 }\end{array}$ \\
\hline $\begin{array}{l}\text { Hospital } \\
\text { website }\end{array}$ & $\begin{array}{l}\text { A } \\
\text { (mean = 4.65) }\end{array}$ & $\begin{array}{l}\text { A } \\
\text { (mean = 4.64) }\end{array}$ \\
\hline Google reviews & $\begin{array}{l}\text { B } \\
\text { (mean = 4.20) }\end{array}$ & $\begin{array}{l}\text { A } \\
\text { (mean = 4.56) }\end{array}$ \\
\hline Vitals & $\begin{array}{l}\text { B } \\
\text { (mean = 4.11) }\end{array}$ & $\begin{array}{l}\text { B } \\
\text { (mean = 4.12) }\end{array}$ \\
\hline HealthGrades & $\begin{array}{l}\text { B } \\
\text { (mean = 3.99) }\end{array}$ & $\begin{array}{l}\text { C } \\
\text { (mean = 3.95) }\end{array}$ \\
\hline RateMDs & $\begin{array}{l}\text { C } \\
\text { (mean = 3.88) }\end{array}$ & $\begin{array}{l}\text { C } \\
\text { (mean = 3.83) }\end{array}$ \\
\hline $\begin{array}{l}\text { Note: Websites not tagged with the same letter in each } \\
\text { column are significantly different. }\end{array}$ \\
\hline
\end{tabular}

In summary, the results of the ANOVA models demonstrated that the means of the scores posted on different PRWs were consistently and significantly different from one another and the highest mean scores belonged to the ratings posted on the hospitals' websites, supporting the hypothesis. Nonetheless, one of the assumptions of ANOVA, namely homogeneity of variance or homoscedasticity, indicates that the groups being compared should have approximately equal variances [8]. Testing this assumption is particularly important in this study because the groups have unequal sample sizes [8]. To test this assumption, we performed Levene's test. The results of the test showed that there were at least two groups (i.e., websites) associated with each hospital that had unequal variances ( $p$-value $<0.001$ ), which is against the homogeneity of variance assumption. To address this issue, we performed two sets of Welch test, which is an appropriate technique for comparisons of data with heterogeneous variances [2]. The results demonstrated that for each hospital, there were still websites with significantly different mean scores ( $p$ value $<0.001$ ), confirming the ANOVA findings.

Nevertheless, the results of the Welch tests did not provide any information about pairwise differences between the websites. Furthermore, ANOVA and Welsh test, inherently, compare the mean values of groups and do not compare the corresponding individual values between groups. Accordingly, they do not compare the physician scores posted on, for example, RateMDs with the scores associated with the exact same physicians posted on the hospitals' websites. To fill these gaps and to further examine the hypothesis, we conducted a series of paired t-tests. To do so, for each hospital, we performed 10 pairwise comparisons between websites for each hospital. In each comparison, we only included the common physicians between the two websites (i.e., the ones that had at least five ratings on both websites). For instance, when comparing the scores posted on hospital_1's website and HealthGrades, we only included 225 physicians who had sufficient ratings on both of those websites.

Table 5: Paired t-test results

\begin{tabular}{|c|c|c|}
\hline $\begin{array}{l}\text { Mean[i] - Mean[j] } \\
\text { Std Err Diff } \\
\text { Sample size }(\mathrm{N})\end{array}$ & Hospital_1 & Hospital_2 \\
\hline $\begin{array}{l}\text { Hospital website - } \\
\text { HealthGrades }\end{array}$ & $\begin{array}{l}0.64 * * * \\
(0.04) \\
N=225\end{array}$ & $\begin{array}{l}0.69 * * * \\
(0.05) \\
\mathrm{N}=172\end{array}$ \\
\hline $\begin{array}{l}\text { Hospital website - } \\
\text { Vitals }\end{array}$ & $\begin{array}{l}0.52 * * * \\
(0.04) \\
N=170 \\
\end{array}$ & $\begin{array}{l}0.43^{* * *} \\
(0.05) \\
\mathrm{N}=132 \\
\end{array}$ \\
\hline $\begin{array}{l}\text { Hospital website - } \\
\text { RateMDs }\end{array}$ & $\begin{array}{l}0.83^{* * *} \\
(0.08) \\
N=68\end{array}$ & $\begin{array}{l}0.80^{* * *} \\
(0.60) \\
\mathrm{N}=37\end{array}$ \\
\hline $\begin{array}{l}\text { Hospital website - } \\
\text { Google Reviews }\end{array}$ & $\begin{array}{l}0.42^{* * *} \\
(0.09) \\
\mathrm{N}=54\end{array}$ & $\begin{array}{l}0.06 \\
(0.09) \\
N=31 \\
\end{array}$ \\
\hline HealthGrades - Vitals & $\begin{array}{l}-0.12^{*} \\
(0.05) \\
\mathrm{N}=147\end{array}$ & $\begin{array}{l}-0.21 * * * \\
(0.06) \\
\mathrm{N}=101\end{array}$ \\
\hline $\begin{array}{l}\text { HealthGrades - } \\
\text { RateMDs }\end{array}$ & $\begin{array}{l}0.15 \\
(0.09) \\
\mathrm{N}=63 \\
\end{array}$ & $\begin{array}{l}0.08 \\
(0.13) \\
\mathrm{N}=31 \\
\end{array}$ \\
\hline $\begin{array}{l}\text { HealthGrades - Google } \\
\text { Reviews }\end{array}$ & $\begin{array}{l}-0.17 \\
(0.10) \\
\mathrm{N}=50\end{array}$ & $\begin{array}{l}-0.48^{* * *} \\
(0.12) \\
\mathrm{N}=29\end{array}$ \\
\hline Vitals - RateMDs & $\begin{array}{l}0.44^{* * *} \\
(0.07) \\
N=54 \\
\end{array}$ & $\begin{array}{l}0.31^{*} \\
(0.11) \\
\mathrm{N}=22 \\
\end{array}$ \\
\hline $\begin{array}{l}\text { Vitals - Google } \\
\text { Reviews }\end{array}$ & $\begin{array}{l}0.01 \\
(0.10) \\
N=40 \\
\end{array}$ & $\begin{array}{l}-0.18 \\
(0.59) \\
N=16 \\
\end{array}$ \\
\hline $\begin{array}{l}\text { RateMDs - Google } \\
\text { Reviews }\end{array}$ & $\begin{array}{l}-0.22 \\
(0.15) \\
\mathrm{N}=21 \\
\end{array}$ & $\begin{array}{l}-0.33 \\
(0.29) \\
\mathrm{N}=9 \\
\end{array}$ \\
\hline
\end{tabular}

For each hospital, four of the pairwise comparisons directly addressed the hypothesis and the remaining six comparisons were performed as additional analysis to understand the extent to which the independent PRWs were different from one another. The results of the paired t-tests for hospital_1 and hospital_2 are presented in Table 5. These results are in line with those of the ANOVA models indicating that 1 ) the highest mean satisfaction scores consistently belonged to the hospital websites, which supported the hypothesis and 2) there were statistically significant 


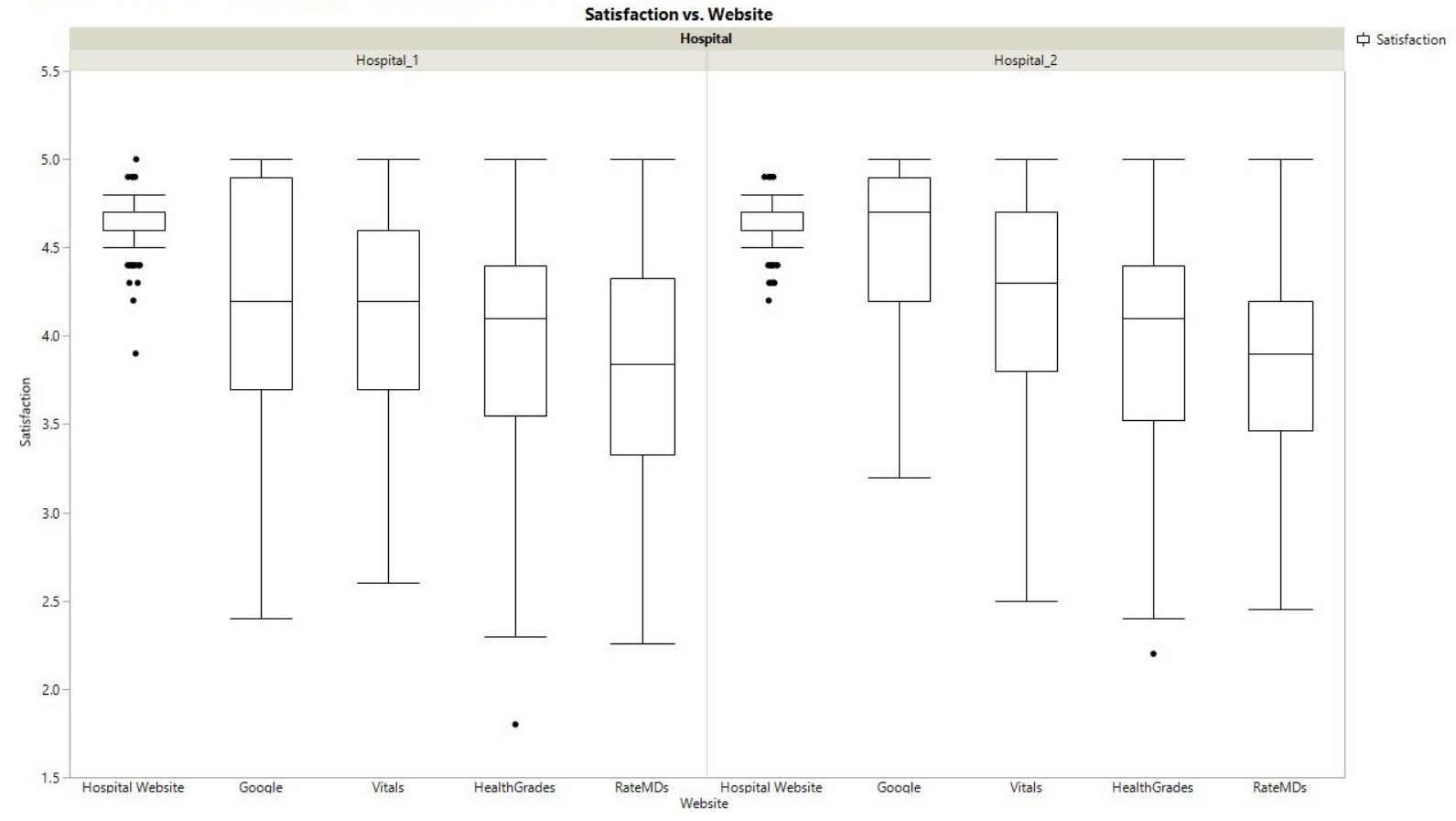

Figure 2: Distribution of patient satisfaction scores for different websites

differences between means of the scores posted on other PRWs. Nonetheless, similar to the ANOVA results, the only non-significant t-test output that belonged to a comparison between a hospital's website and an independent PRW was associated with hospital_2 and Google Reviews. However, that difference is still marginally significant ( $p$-value < 0.1 ), which is consistent with the hypothesis.

Finally, we used box plots to visually examine the distributions of ratings published on the hospitals' websites and compare those distributions with the corresponding ones associated with the independent PRWs. Box plots, in general, show a compact view of a variable's distribution (in this case satisfaction scores) with quartiles [22]. Thus, they are great tools to understand whether the values of a variable are notmuch deviated from the mean and highly skewed toward high or low values of that variable.

The results of our box plot analysis, presented in Figure 2, showed that for the both hospitals, the ratings had much lower variations on the hospitals' websites compared to those on the independent PRWs. These findings are consistent with the results of the Welsh tests conducted earlier in this study. Moreover, the low variations of scores on the hospitals' websites confirmed the hypothesis of more significant bias in the ratings published on healthcare organizations' websites. The reason is that a significantly higher mean value combined with a much narrower distribution of ratings on healthcare organizations' websites implies that even those physicians who have low ratings on independent PRWs have high ratings on the organization-administered websites.

\section{Discussion}

PRWs are intended to be used as reliable sources of information to assess the quality of care that physicians offer to their patients. However, prior research has shown that, for different reasons, the ratings and reviews posted on PRWs are to some extent biased toward positive values. In this study, we demonstrated that the inaccuracy and bias toward positive values in online physician ratings posted on healthcare organizations' websites were more intense than those posted on independent PRWs. This high level of inaccuracy may mislead Internet users in their decision making on which healthcare providers to choose. The reason is that individuals who search for the reviews and ratings of physicians on the Internet may first see the healthcare organization's website and rely on the information provided to them on those websites. Thus, the online information seekers in this context may not even get to look at other PRWs to find additional reviews and performance information about physicians.

Consistent with our findings, prior research has shown that individuals are becoming more and more suspicious of the ratings posted on healthcare websites. For instance, Holliday et al. [12] found that $57 \%$ of the patient respondents in their study expressed a high level of trust and perceived accuracy of numerical ratings and narrative comments published on independent PRWs; whereas, only 43\% of them had positive attitudes toward similar information that were published on healthcare organizations' websites. Interestingly, most of the physician respondents in that study believed that the 
accuracy and trustworthiness of rating information published on the providers' websites were higher than those provided by independent PRWs.

The results of our study showed that, from a practical standpoint, healthcare providers should investigate the reason(s) of the significantly higher patient satisfaction scores on their websites. Once they find the reason(s), they should accordingly, try to eliminate the systematic mechanisms and procedures that are causing such high levels of bias. In this way, those organizations will gain trust and ultimately, enhance the usability and reliability of their physician rating platforms. Also, on the users' side, those individuals who frequently use PRWs to compare the quality of care that doctors offer should be aware of such biases and ensure that they use different PRWs including those provided by healthcare organizations as well as the independent ones - to collect, combine, compare, and contrast the rating information provided on those websites and accordingly, make a more informed decision for their healthcare. From a theoretical standpoint, the results of this study supported and extended the findings of prior studies (e.g., $[4,5,14])$ that have collectively, indicated that online physician ratings are positively biased and may not accurately reflect physicians' quality of care.

This study has limitations. First, we only analyzed the ratings associated with the physicians in two hospitals, which were both operated by the same health system. Future research should examine a wide range of hospitals operating under different health systems in different states, regions, and possibly countries to further address the issue of bias in this context. Second, in the current study, we only focused on four independent PRWs; whereas, there are several other such websites that are being used in the United States and in other countries. Future research can address this limitation by collecting and analyzing data from other independent PRWs. Third, we did not investigate the actual information filtration procedures that healthcare organizations employ to decide on whether or not to remove a physician rating or review from their websites. Researchers in future studies can interview the companies that handle physician rating surveys for healthcare organizations to see if their information assurance and filtration mechanisms are a major source of inflated bias in that context. This will help us understand what the actual underlying reasons behind these biases are.

\section{Conclusion}

In this study, we examined the differences between physician ratings in terms of patient satisfaction scores published on healthcare organizations' websites and the ones published on independent PRWs. The results demonstrated that the ratings published on healthcare organizations' websites were significantly higher than the corresponding scores published on the independent PRWs. Moreover, our findings showed that the variations of the ratings on the providers' websites were significantly lower than those on the PRWs. These findings supported a high level of bias toward positive values in the ratings administered by healthcare organizations and published on their websites. Accordingly, Internet users should be aware of such systematic biases. Moreover, the results implied that healthcare providers should feel more responsible about the rating information that they post on their websites as that information may ultimately be used by individuals to make decisions for their healthcare. Therefore, healthcare organizations should put more effort into assuring that the ratings and reviews published on their websites are, at least, not significantly more biased than similar information posted on independent PRWs.

\section{References}

[1] Bakhsh, W., \& Mesfin, A. "Online ratings of orthopedic surgeons: analysis of 2185 reviews”, The American Journal of Orthopedics, 43(8), 2014, pp. 359-363.

[2] Brown, M. B., \& Forsythe, A. B. “372: the ANOVA and multiple comparisons for data with heterogeneous variances”, Biometrics, 1974, pp. 719-724.

[3] Ellimoottil, C., Hart, A., Greco, K., Quek, M. L., \& Farooq, A. "Online reviews of 500 urologists", The Journal of Urology, 189(6), 2013, pp. 2269-2273.

[4] Emmert, M., \& Meier, F. “An analysis of online evaluations on a physician rating website: evidence from a German public reporting instrument”, Journal of Medical Internet Research, 15(8), 2013, e157.

[5] Gao, G. G., Greenwood, B. N., Agarwal, R., \& McCullough, J. S. "Vocal minority and silent majority: how do online ratings reflect population perceptions of quality?”, MIS Quarterly, 39(3), 2015, pp. 565-589

[6] Gao, G. G., McCullough, J. S., Agarwal, R., \& Jha, A. $\mathrm{K}$. "A changing landscape of physician quality reporting: analysis of patients' online ratings of their physicians over a 5-year period”, Journal of Medical Internet Research, 14(1), 2012, e38.

[7] Gastwirth, J. L., Gel, Y. R., \& Miao, W. "The impact of Levene's test of equality of variances on statistical theory and practice”, Statistical Science, 2009, pp. 343-360.

[8] Glass, G. V., Peckham, P. D., \& Sanders, J. R. "Consequences of failure to meet assumptions underlying 


\section{51st Hawaii International Conference on System Sciences}

the fixed effects analyses of variance and covariance”, Review of Educational Research, 42(3), 1972, pp. 237-288.

[9] Grabner-Kräuter, S., \& Waiguny, M. K. "Insights into the impact of online physician reviews on patients' decision making: randomized experiment”, Journal of Medical Internet Research, 17(4), 2015, e93.

[10] Hanauer, D. A., Zheng, K., Singer, D. C., Gebremariam, A., \& Davis, M. M. "Public awareness, perception, and use of online physician rating sites", The Journal of the American Medical Association, 311(7), 2014, pp.734-735.

[11] Hao, H. "The development of online doctor reviews in China: an analysis of the largest online doctor review website in China”, Journal of Medical Internet Research, 17(6), 2015, e134.

[12] Holliday, A. M., Kachalia, A., Meyer, G. S., \& Sequist, T. D. "Physician and patient views on public physician rating websites: a cross-sectional study” Journal of General Internal Medicine, 2017, pp. 1-6.

[13] Jha, A. K. "Health Care Providers Should Publish Physician Ratings", Harvard Business Review, October 2015.

[14] Kadry, B., Chu, L. F., Kadry, B., Gammas, D., \& Macario, A. "Analysis of 4999 online physician ratings indicates that most patients give physicians a favorable rating”, Journal of Medical Internet Research, 13(4), 2011, e95.

[15] Kordzadeh, N. (2016). "Social Media in Health Care" In Contemporary Consumer Health Informatics, pp. 101123: Springer.

[16] Kordzadeh, N., \& Young, D. "Understanding How Hospitals Use Social Media: An Exploratory Study of Facebook Posts”, Proceedings of the Americas Conference on Infomration Systems, San Juan, Puerto Rico, 2015.

[17] Lagu, T., Hannon, N. S., Rothberg, M. B., \& Lindenauer, P. K. "Patients' evaluations of health care providers in the era of social networking: an analysis of physician-rating websites”, Journal of General Internal Medicine, 25(9), 2010, pp. 942-946.

[18] Lagu, T., Metayer, K., Moran, M., Ortiz, L., Priya, A., Goff, S. L., \& Lindenauer, P. K. "Website characteristics and physician reviews on commercial physician-rating websites", The Journal of the American Medical Association, 317(7), 2017, pp. 766-768.

[19] Lawler, C. "Duluth Doctor Appealing Judge's Decision to Toss out Defamation Suit”, Duluth News Tribune, 2011.

[20] Mackay, B. "RateMDs.com nets ire of Canadian physicians”, The Canadian Mesical Association Journal,
17(6), 2007, Retrieved from

http://www.cmaj.ca/content/176/6/754

[21] Magaw, T. "Cleveland Clinic believes posting ratings of physicians is a healthy step", 2015, Retrieved from http://www.crainscleveland.com/article/20150621/NEWS/1 50619731/cleveland-clinic-believes-posting-ratings-ofphysicians-is-a-healthy

[22] McGill, R., Tukey, J. W., \& Larsen, W. A. "Variations of box plots", The American Statistician, 32(1), 1978, pp. 12-16.

[23] n.d. “'I'm not too happy with this doctor': Hospitals post physician reviews on their websites”, 2014, Retrieved from https://www.advisory.com/dailybriefing/2014/04/18/hospitals-post-doctor-reviews-on-theirwebsite

[24] Ramey, C. "Long Island Hospital Posts Doctor Ratings”, 2015, Retrieved from

https://www.wsj.com/articles/long-island-hospital-postsdoctor-ratings-1440635377

[25] Raphael, J. "Posting Negative Reviews Could Get You Sued”, PC World, 2009.

[26] Terlutter, R., Bidmon, S., \& Röttl, J. "Who uses physician-rating websites? Differences in sociodemographic variables, psychographic variables, and health status of users and nonusers of physician-rating websites”, Journal of Medical Internet Research, 16(3), 2014, e97. 